\title{
Tomography of photoelectron distributions produced through strong-field photodetachment of $\mathrm{Ag}^{-}$
}

\author{
Mikael Eklund $\odot$, Hannes Hultgren, Igor Kiyan,* and Hanspeter Helm $\odot$ \\ Physikalisches Institut, Albert-Ludwigs-Universität, 79104 Freiburg, Germany \\ Dag Hanstorp $\dagger$ \\ Department of Physics, University of Gothenburg, SE-412 96 Gothenburg, Sweden
}

(Received 20 February 2020; accepted 7 July 2020; published 19 August 2020)

\begin{abstract}
We have applied a tomographic imaging method to recreate the full three-dimensional distribution of photoelectrons produced in a strong-field photodetachment process. The method is general and can be applied to any laser polarization. This stands in contrast to traditional imaging inversion methods, such as Abel inversion, which require prior knowledge of the symmetries of the electron distribution and are therefore limited to experiments using linear or circular polarization. Our method is also useful in a situation where linear polarization is used, since it compensates for detector inhomogeneities by spreading the information on a larger detector surface. In addition, it facilitates a method to detect polarization defects. Measurements were made for photodetachment of $\mathrm{Ag}^{-}$at laser wavelengths of 1310 and $2055 \mathrm{~nm}$, and were found to agree well with simulations in the strong field approximation. The data in the $1310 \mathrm{~nm}$ case revealed an unexpected asymmetry in the plane in which the laser polarization axis is rotated. Using a quasistationary quasienergy state model, a residual elliptical polarization of $\varepsilon=0.21 \pm 0.01$ consistently explains the observed asymmetry. We conclude that the method described in this paper has the potential to be applied in experiments where a more complete characterization of electron emission distributions is required.
\end{abstract}

DOI: 10.1103/PhysRevA.102.023114

Electron imaging has become an ubiquitous tool to record the momentum and angular distribution of electrons emitted in photoionization and photodetachment processes [1]. Such imaging methods are also used for recording the distributions of heavy charged particles which emerge from laser-induced processes [2]. In its simplest implementation, the imaging method relies on projecting the emerging charged particles onto a planar detector using an external electric field [3]. Linearly polarized light produces a cylindrically symmetric spatial distribution around the polarization axis. For circularly polarized light, on the other hand, a cylindrically symmetric spatial distribution appears around the laser propagation axis. In both cases, a position-sensitive detector located in a plane parallel to these selected axes can be used to record an image of charged particle impacts which is twofold symmetric, with one symmetry axis along the selected axis of projection and one along an axis perpendicular to it. When the kinetic energy gained in the projection field is much larger than the excess energy from the photo process, it is possible to apply an

\footnotetext{
*Present address: Helmholtz-Zentrum Berlin, Hahn-Meitner-Platz 1, 14109 Berlin, Germany.

${ }^{\dagger}$ Corresponding author: dag.hanstorp@gu.se

Published by the American Physical Society under the terms of the Creative Commons Attribution 4.0 International license. Further distribution of this work must maintain attribution to the author(s) and the published article's title, journal citation, and DOI. Funded by Bibsam.
}

inversion routine, such as the inverse Abel transform (IAT) [4], to transform the 2D projection image on the detector into 3D information of the spatial electron distribution in the atomic or molecular frame.

In the general case, however, a more elaborate analysis is required. This is, for example, the case when elliptically polarized light is used, or in experiments aimed at a complete characterization of photoionization [5]. A similar situation arises in experiments where the photoelectron energy is affected by different pondermotive acceleration parallel and perpendicular to the laser beam [6]. Furthermore, when electron emission is preferentially parallel to the laser polarization (such as is generally the case in strong electromagnetic fields), conventional inversion routines produce distortions since single-image inversion causes an accumulation of inversion errors along the axis of symmetry. There are a number of alternatives to the Abel inversion such as BASEX or pBASEX but they all require that there is a known symmetry axis. For a comparison of performance, speed, and noise accumulation of different inversion methods requiring a symmetry axis, see Refs. [7-10]. A more elaborate analysis requires images to be recorded at different orientations of the polarization vector. In its most general implementation, this can be achieved by a tomographic reconstruction [11-13]. For example, Wollenhaupt et al. [14] recreated the three-dimensional photoeectron distribution produced through resonantly enhanced multiphoton photoionization of potassium. Smeenk et al. [15] used a similar technique to obtain the distribution of photoelectrons produced through tunnel ionization of argon. The method of tomographic reconstruction has been further discussed by Wollenhaupt et al. [16] and Hocket et al. [17,18]. 
In the present paper, we build on the methods for photoelectron tomography of Wollenhaupt et al. [14] and Smeenk et al. [15] and apply it to photodetachment of negative ions in a fast beam. The ultimate goal is to combine our pump-probe technique $[19,20]$ with a tomographic method for the purpose of extracting more accurate information about the state of alignment of the atom than is available in traditional photoelectron imaging measurements. We apply our tomographic method to minimum two- and three-photon detachment of $\mathrm{Ag}^{-}$ions for which theoretical model calculations can be performed. This enables us to compare the calculations on a one-to-one basis with the experimental data.

Our results show an unexpected feature in an asymmetry of the photoelectron distribution in the polarization plane of the laser field. We explain the asymmetry as a result of a residual elliptical polarization of the laser. In a traditional photoelectron imaging experiment, this asymmetry would not be visible, which shows that the sensitivity of the tomographic method makes it also useful in cases where standard imaging methods are normally employed.

\section{EXPERIMENTAL SETUP}

The experimental setup is described in detail elsewhere [21]. In short, $\mathrm{Ag}^{-}$ions are formed in a cesium sputter source, accelerated to $4.5 \mathrm{keV}$, and mass selected in a Wien filter. Einzel lenses are used to focus the ion beam in the interaction region, where the beam waist is $1 \mathrm{~mm}$ at a flux of $27 \mathrm{nA}$. A quadrupole deflector serves to separate, by $90^{\circ}$, the negative-ion beam from neutral atoms formed by collisional detachment in the beam path prior to the interaction region. Three differentially pumped sections maintain a residual pressure of the order of $10^{-9}$ mbar in the interaction region. The laser pulses, generated in an optical parametric amplifier pumped by a Ti:sapphire laser system at a repetition rate of $1 \mathrm{kHz}$, have wavelengths of 1310 and $2055 \mathrm{~nm}$ and pulse energies of 60 and $30 \mu \mathrm{J}$, respectively. The signal and idler are separated by means of a dichroic mirror. Either the signal or the idler is directed to the electron spectrometer. In both cases, the pulse length is $130 \mathrm{fs}$ full width at half maximum (FWHM). The laser beam is focused by a lens of $20-\mathrm{cm}$ focal length and crosses the ion beam perpendicularly. The intensity distribution at the laser focus is measured by scanning a razor blade across the focal region. The shape of the pulses can be described well by a Gaussian profile with a waist of 27 and $44 \mu \mathrm{m}$ FWHM, yielding peak intensities in the laser focus of the order of $5.3 \times 10^{13} \mathrm{~W} / \mathrm{cm}^{2}$ and $9.6 \times 10^{12} \mathrm{~W} / \mathrm{cm}^{2}$ for the 1310- and 2055-nm pulses, respectively.

The central part of the setup is an electron imaging spectrometer, which projects electrons emitted in the entire solid angle onto a two-dimensional position-sensitive detector by an electrostatic field. The position-sensitive detector consists of two image-quality micro-channel plates in chevron configuration and a phosphor screen. The light from the phosphor screen is recorded by a CCD array with 12-bit resolution where it is accumulated and read out to a computer at intervals of a few seconds. The electron-imaging spectrometer is operated in the velocity mapping regime [3]. The background caused by collisional detachment is reduced by pulsing the ion beam with $10 \mu \mathrm{s}$ on and $990 \mu \mathrm{s}$ off for each laser shot period.

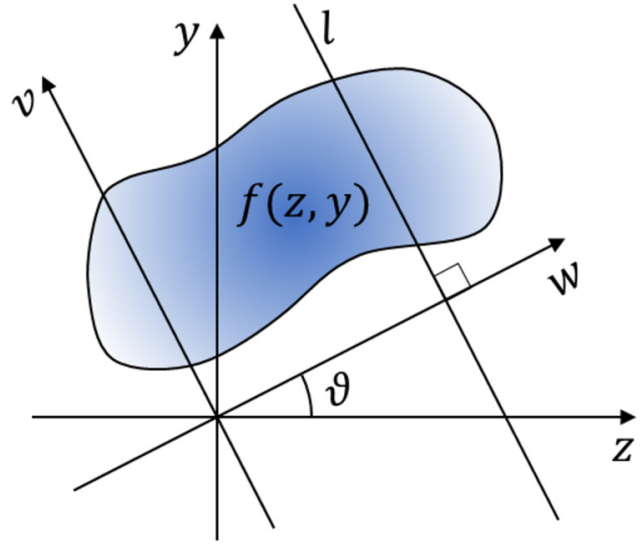

FIG. 1. Variables needed for the Radon transform. The $z$ axis is defined by the laser (major) polarization axis. The $w$ and $v$ axes are parallel to the detector surface and the projection field, respectively.

This switching also acts as an additional mass filter due to the different times of flight of different atomic and molecular species. Additionally, the voltage over the MCPs is reduced in the period between laser pulses to further reduce background noise. Under typical operating conditions, about five events per laser shot are recorded. Distortions by space charge effects do not occur at such low electron yields.

Images suitable for tomography are obtained by rotating the laser polarization in step sizes of $3^{\circ}$ using a stepper motor-driven half-wave plate (Thorlabs AHWP05M-1600) and recording images for 25000 laser shots at each angular position of the half-wave plate. A tomographic scan could therefore be recorded in about 30 minutes. During this time period, the laser power, pointing stability, and ion beam density were essentially stable.

\section{INVERSION METHOD}

Mathematically, the projection of a two-dimensional object onto a set of straight lines is described by the two-dimensional Radon transform. Let $f(z, y)$ be a continuous function on a two-dimensional space $\mathbf{R}^{2}$ with compact support and let $\mathbf{L}$ be the space of straight lines in $\mathbf{R}^{2}$. Then for all $l \in \mathbf{L}$ there exists a parametrization by $v$ as

$$
\begin{aligned}
l= & \left\{(z(v), y(v)) \in \mathbf{R}^{2} \mid(v \sin \vartheta+w \cos \vartheta,\right. \\
& -v \cos \vartheta+w \sin \vartheta)\},
\end{aligned}
$$

where

$$
-\infty<w<\infty
$$

and

$$
0 \leqslant \vartheta<\pi
$$

Here $w$ and $\vartheta$ are coordinates in $\mathbf{L}$ where $w$ is the perpendicular distance to the line from the origin and $\vartheta$ is the angle this distance vector makes with the $z$ axis (see Fig. 1). 


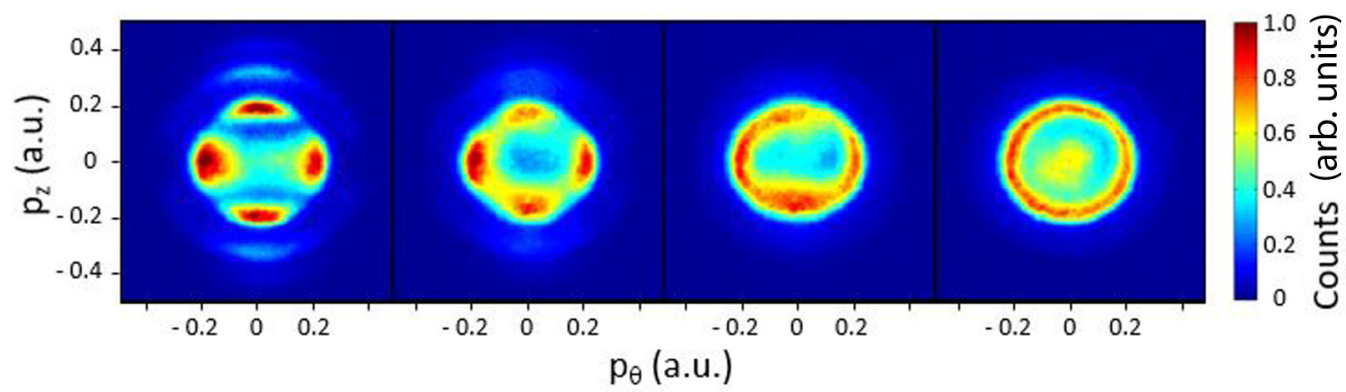

FIG. 2. Relative photoelectron momentum distribution of projections recorded for photodetachment at $1310 \mathrm{~nm}$ at angles of $\vartheta=0^{\circ}, 30^{\circ}$, $60^{\circ}$, and $90^{\circ}$. A video showing the series of projections for a full rotation at steps of $3^{\circ}$ is shown in the Supplemental Material [27].

With this parametrization, the Radon transform $R$ is the transform $R: \mathbf{R}^{2} \rightarrow \mathbf{L}$ defined as

$$
\begin{aligned}
& (R f)(w, \vartheta)= \\
& =\int_{-\infty}^{\infty} f((v \sin \vartheta+w \cos \vartheta),(-v \cos \vartheta+w \sin \vartheta)) \mathrm{d} v
\end{aligned}
$$

This is the integral of $f$ along the straight line defined by $w$ and $\vartheta$. A number of methods exist to perform the inverse Radon transform (IRT), and thus recreate the threedimensional distribution. An algebraically concise expression for the IRT is given in Ref. [22] as

$$
\begin{aligned}
f(z, y)= & \frac{1}{\pi} \lim _{c \rightarrow 0} \int_{0}^{\pi} \int_{-\infty}^{\infty}(R f) \\
& \times(w-z \cos \vartheta-y \sin \vartheta, \vartheta) G_{c}(w) \mathrm{d} w \mathrm{~d} \vartheta
\end{aligned}
$$

where the convolution kernel $G_{c}$ is given by

$$
G_{c}(w)= \begin{cases}\frac{1}{\pi c^{2}}, & |w| \leqslant c \\ \frac{1}{\pi c^{2}}\left(1-\frac{1}{\sqrt{1-c^{2} / w^{2}}}\right), & |w|>c .\end{cases}
$$

A direct use of Eq. (3) for inversion of discrete projections is not possible, however, and other methods are required. Wollenhaupt et al. [14] used a method based on the mathematical equivalence of the one-dimensional Fourier transform of the Radon transform making a slice through the two-dimensional Fourier transform. This is a consequence of the projectionslice theorem [23,24]. Smeenk et al. [15] instead used a filtered backprojection scheme where a Shepp-Logan filter was applied to suppress inversion artifacts. In the present paper, we have also opted to use the filtered backprojection method, but using a Hamming filter instead. The choice of the Hamming filter was made, as this was found to perform well in the case of a smooth distribution. Owing to its frequent use in medical applications, stable numerical implementations of the filtered backprojection are readily available. In our case, we used a routine available in MATLAB [25].

In our experiment, the laser polarization semimajor axis is aligned along the $z$ axis and the $w$ axis lies in the detector plane. The $x$ axis is defined to be along the laser propagation. This means that we record images in the $(x, w)$ plane for a series of $\vartheta$ values so every column (i.e., $x$ position) in the recorded images represent a two-dimensional Radon transform of a slice of the electron swarm. In fact, in the case when the electron swarm has cylindrical symmetry around the $z$ axis, the central column of the recorded images contains sufficient information to recreate the full electron distribution. In practice, however, this is not always feasible as the count rate for any single column is relatively low under typical experimental conditions.

\section{DEMONSTRATION OF TOMOGRAPHY}

In this section, we present experimental data obtained from photodetachment of $\mathrm{Ag}^{-}(\mathrm{EA}(\mathrm{Ag})=1.30447 \mathrm{eV}$ [26] $)$ as well as a comparison of the results to a theoretical model. These results are then used to demonstrate the tomographic reconstruction. Measurements were made at laser wavelengths of $1310 \mathrm{~nm}(h v=0.9464 \mathrm{eV})$ and $2055 \mathrm{~nm}(h v=0.6033 \mathrm{eV})$. At these wavelengths, the lowest order photodetachment channel involves absorbtion of two and three photons, respectively.

Figure 2 shows examples of projections recorded for a laser wavelength of $1310 \mathrm{~nm}$ at angles of $\vartheta=0^{\circ}, 30^{\circ}, 60^{\circ}$, and $90^{\circ}$. The full series of projections with steps of $3^{\circ}$, shown in the Supplemental Material [27], is used to recreate the 3D photoelectron distribution. Three isosurfaces, corresponding to relative photoelectron yields of 0.1 (blue), 0.2 (green), and 0.4 (brown) of the maximum value of this distribution, are shown in Fig. 3. The left panel shows the front view where the laser propagation axis and the laser polarization axis represent the directions of the (negative) horizontal and vertical axes of the image, respectively. The right panel shows a side view where the laser propagation axis is perpendicular to the figure and the laser polarization axis points in the vertical direction. It can be seen in the right panel that the torus produced by two-photon detachment comes out at an angle with respect to the axis on which the caps of the two- and three-photon detachment lie, which deviates from the expected $90^{\circ}$. This breaks the cylindrical symmetry of the electron swarm. Possible explanations of this tilt will be addressed in the following section. Figure 4 shows the same views for $2055 \mathrm{~nm}$. In this case, an asymmetry does not appear in the right panel. The left-right asymmetry in the left panels, for both wavelengths, can be ascribed to inhomogeneities in the detector response.

\section{COMPARISON TO THE STRONG FIELD APPROXIMATION FOR LINEARLY POLARIZED LIGHT}

Theoretical electron distributions were simulated in the strong-field approximation as described by Gribakin and Kuchiev [32]. The simulation procedure is described in detail 

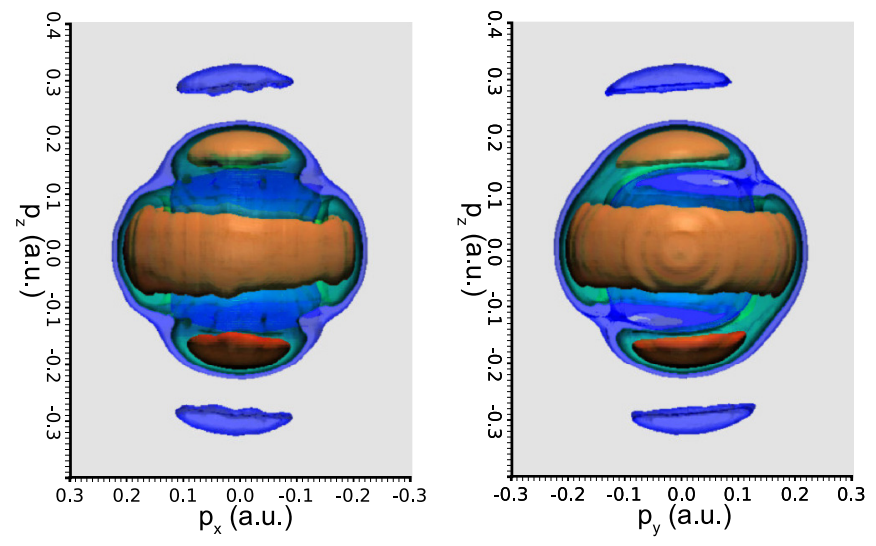

FIG. 3. Reconstructed three-dimensional photoelectron momentum distribution for detachment of $\mathrm{Ag}^{-}$at $1310 \mathrm{~nm}$. The figure shows three isosurfaces at values 0.4 (brown), 0.2 (green), and 0.1 (blue) of the maximum photoemssion distribution value. The laser is propagating in the $x$ direction and the laser polarization is along the $z$ axis. The full reconstructed momentum distribution is shown in the Supplemental Material in movie 2 (straight projection) [28] and movie 3 (slanted projection) [29].

in Ref. [21]. Figures 5 and 6 show a comparison between theory and experiment for $1310 \mathrm{~nm}$ and $2055 \mathrm{~nm}$, respectively. The figures show the angle-resolved electron momentum distribution which is obtained by integrating the full 3D distribution along the azimuthal angle $\phi=\arctan \left(p_{x} / p_{y}\right)$.

In the $1310 \mathrm{~nm}$ case, the experiment shows a clear excess photon detachment (EPD) structure with two-, three-, and four-photon detachment signals at momenta $p \approx 0.187$, 0.311 , and 0.397 . Theory reproduces all the main features of the experiment although it overestimates the three-photon detachment yield along the polarization axis. In the $2055 \mathrm{~nm}$ case in Fig. 6, the experimental electron distribution shows more pronounced higher order EPD peaks due to the lower
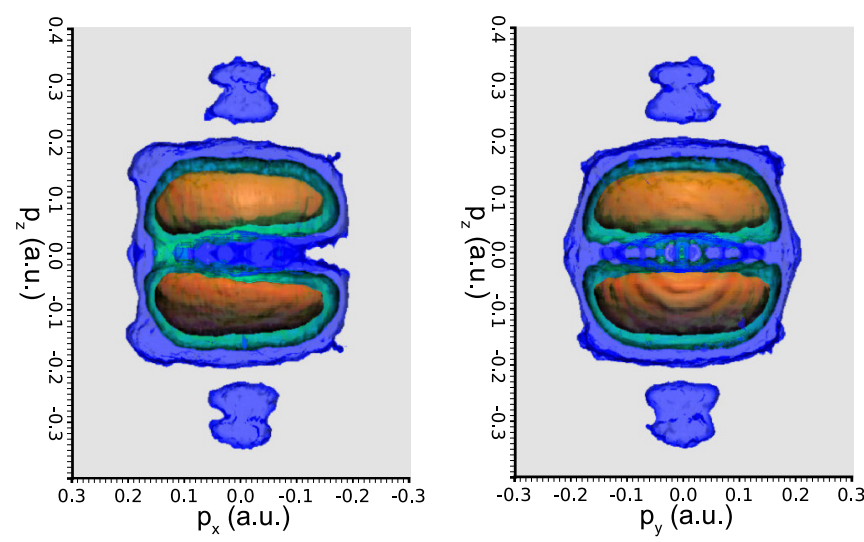

FIG. 4. Reconstructed three-dimensional photoelectron momentum distribution for detachment of $\mathrm{Ag}^{-}$at $2055 \mathrm{~nm}$. The figure shows three isosurfaces at values 0.4 (brown), 0.2 (green), and 0.1 (blue) of the maximum photoemission distribution value. The laser propagates along the $x$ direction and the laser polarization is along the $z$ axis. The full reconstructed momentum distribution is shown in the Supplemental Material in movie 4 (straight projection) [30] and movie 5 (slanted projection) [31].

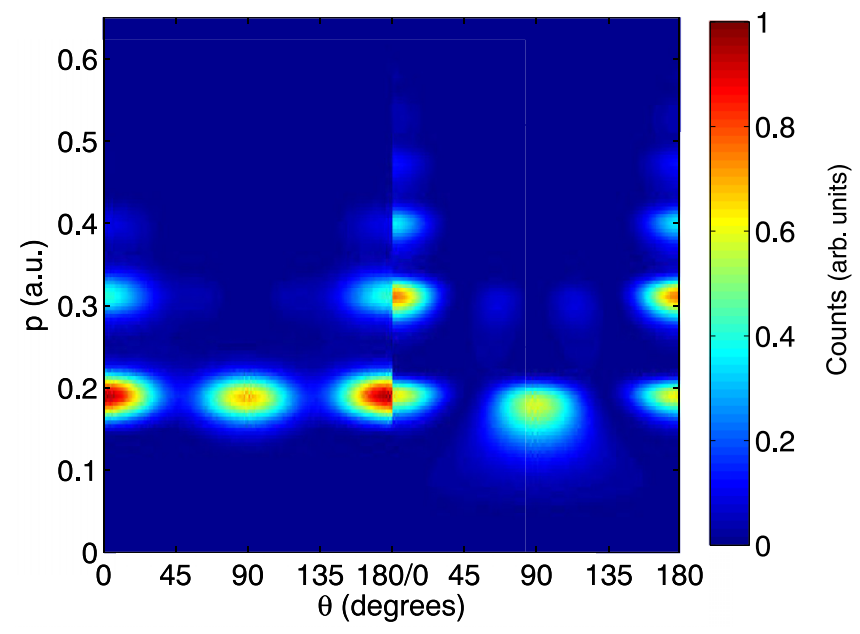

FIG. 5. Comparison of the experiment (left) and the theory of Gribakin and Kuchiev (right) of the relative photoelectron momentum distribution for detachment of $\mathrm{Ag}^{-}$at $1310 \mathrm{~nm}$. The figure is in polar coordinates where $\theta$ is the angle between the electron momentum and the laser polarization axis.

detachment rate, allowing the ions to survive to higher intensities. Here theory also reproduces the main features in the experiment. The simulation procedure takes the spatiotemporal intensity distribution into account. The theoretical electron distribution is subject to the same processing as the experimental data to make sure that any distortion induced by the inversion is also present in the simulated data. The only noticeable inversion artifact is the slight distortion at an emission angle of $45^{\circ}$ and $135^{\circ}$.

\section{COMPARISON TO QQES THEORY FOR ELLIPTICAL POLARIZATION}

It is possible to rule out imperfect projection of photoelectrons, detector inhomogeneities, as well as the inversion

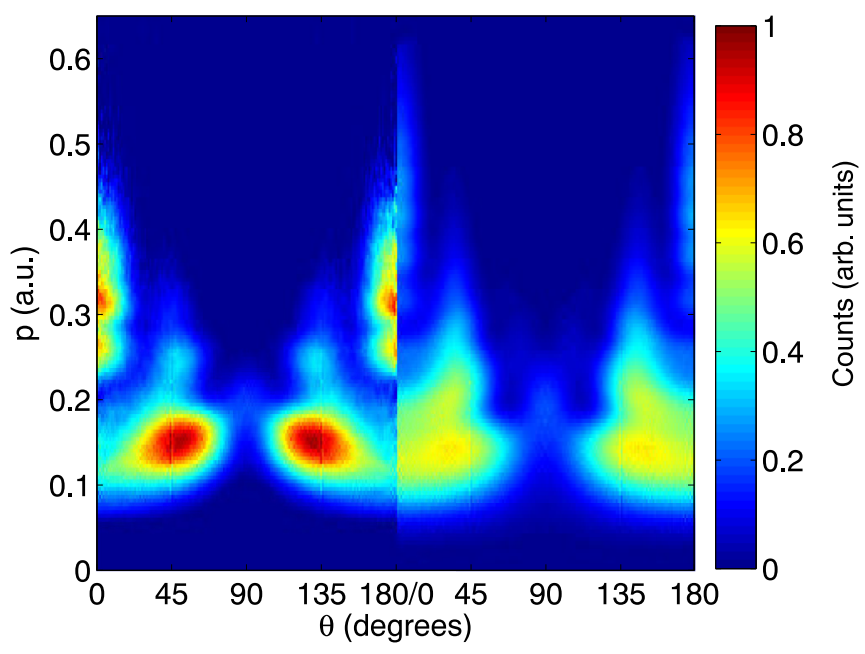

FIG. 6. Comparison of the experiment (left) with the theory of Gribakin and Kuchiev (right) of the relative photoelectron momentum distribution for detachment of $\mathrm{Ag}^{-}$at $2055 \mathrm{~nm}$. The figure is in polar coordinates where $\theta$ is the angle between the electron momentum and the laser polarization axis. 


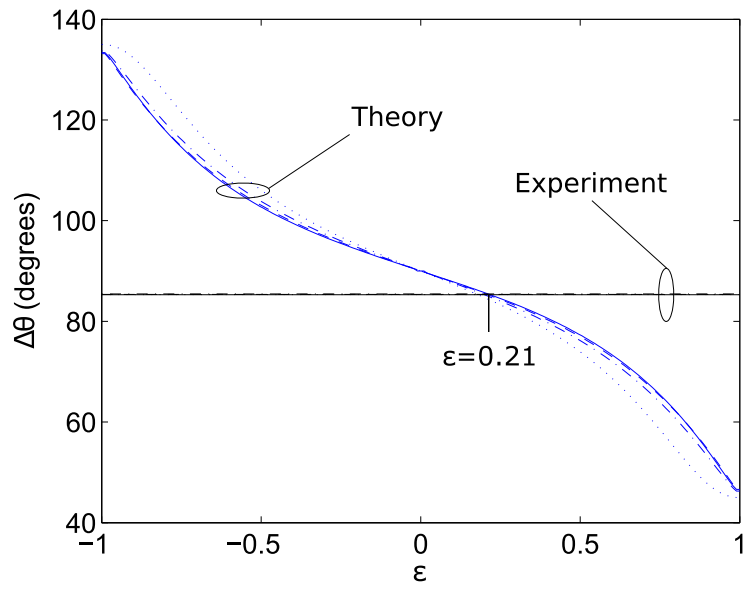

FIG. 7. The angular distance between the polar and equatorial maxima for laser field strengths of 1.5 (solid), 3.0 (dashed), 4.5 (dash-dotted), and 6.0 (dotted) in units of $10^{-3}$ a.u. for two-photon detachment of $\mathrm{Ag}^{-}$at $1310 \mathrm{~nm}$. Blue curves represent simulated values as a function of the ellipticity $\varepsilon$ and the black curves represent the experimentally measured values.

method as causing factors of the asymmetry observed in Fig. 3 since the image acquisition is performed over a full rotation around the laser propagation axis, thereby averaging out any asymmetry. The most plausible explanation for the asymmetry is a residual elliptical polarization of the laser beam. Only through simulations using an elliptical polarization can the observed asymmetry be reproduced. Since the theory of Gribakin and Kuchiev only applies to linear polarization, it is necessary to use an alternate theoretical approach. One powerful method that is capable of explaining the asymmetry in terms of a residual elliptical polarization is the quasistationary quasienergy state approach (QQES) [33]. In the QQES approach, the time-dependent Schrödinger equation (SE) is rewritten to take the form of a stationary SE. In addition to this, the results have the property of being gauge invariant, a property absent in Keldish/Faisal/Reiss theories [34-36]. The code for calculating the quasienergy and angular distribution was obtained from the authors of Ref. [33]. Using the simulation procedure described in Ref. [21] the electron distribution in the polarization plane was simulated taking the spatio-temporal population of the laser focal region into account. Due to the significant computational demands of the QQES method, the simulation was limited to the two- and three-photon processes.

To be able to estimate the ellipticity of the laser polarization in the experiment, the angular distance $\Delta \theta$ between the polar (close to the $p_{z}$ axis) and equatorial (close to the $\left(p_{x}, p_{y}\right)$ plane) maxima was used as a metric. $\Delta \theta$ was determined by measuring the distance between the maxima of the polar cap and the equatorial ring at four different radii, yielding an average value of $\Delta \theta=85,5+/-0,7$ degrees.

Figure 7 shows the relative angular distance between the maxima as a function of the ellipticity $\varepsilon$. Blue curves represent the simulated angular distance between the polar and equatorial maxima distributions for the field strengths $F=$ $\{1.5,3.0,4.5,6.0\} \times 10^{-3}$ a.u. and are shown to intersect with the experimentally determined angle (black) at an ellipticity of

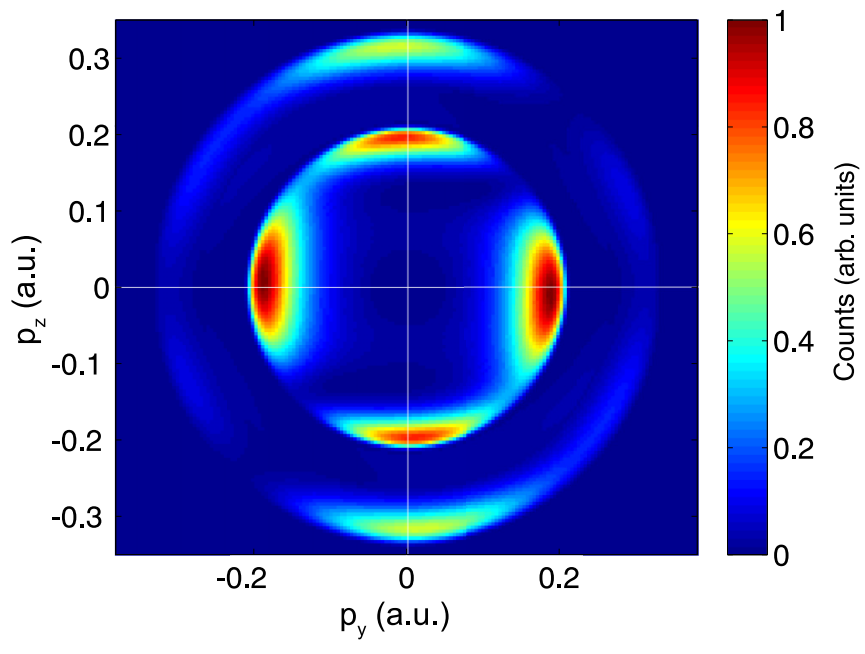

FIG. 8. Simulated relative photoelectron momentum distribution in the central polarization plane obtained through simulation of twoand three-photon detachment of $\mathrm{Ag}^{-}$at a wavelength of $1310 \mathrm{~nm}$, using the QQES method with an ellipticity of $\varepsilon=0.21$.

$\varepsilon=0.21 \pm 0.01$. The simulation for this estimated ellipticity is shown in Fig. 8 and the experimental distribution is shown in Fig. 9. As was the case for the Gribakin and Kuchiev simulation in Fig. 5, the three-photon yield is overestimated in the simulation compared to the yield of the two-photon process, but to a smaller degree. At first glance, Figs. 8 and 9 appear almost symmetric. To emphasize the lack of mirror symmetry, we show in Figs. 10 and 11 the contour plots of the photoelectron yield in the polarization plane for the experimental and simulated distributions, respectively. To de-emphasize the ratio between the yields of the twoand three-photon processes, the yields have been individually normalized. The angular distributions for the experimental and simulated images are in good agreement for both the twoand three-photon processes and the asymmetry seen in the experimental data is reproduced in the simulation. The inset in

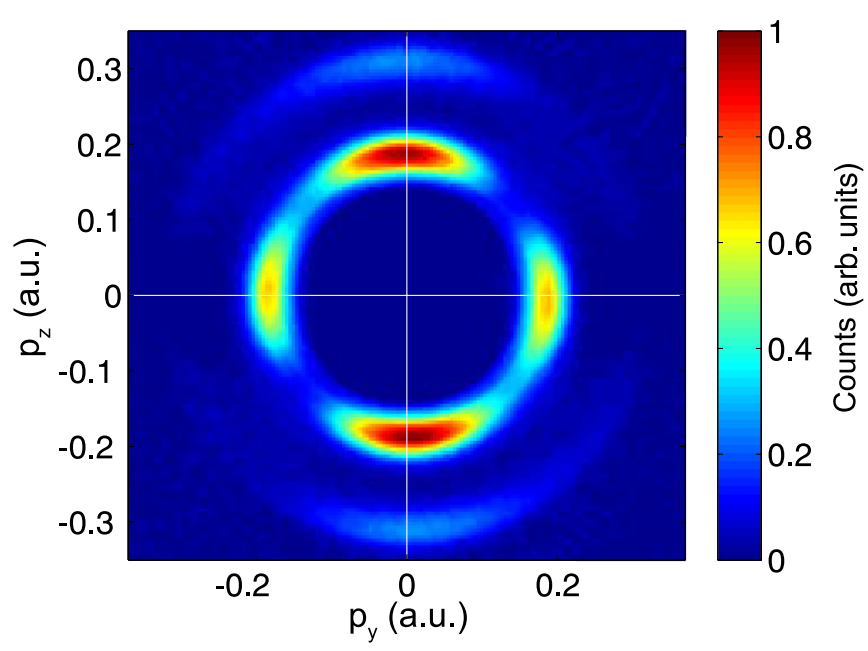

FIG. 9. Experimental relative photoelectron momentum distribution in the central polarization plane obtained for two- and threephoton detachment of $\mathrm{Ag}^{-}$at a wavelength of $1310 \mathrm{~nm}$. 


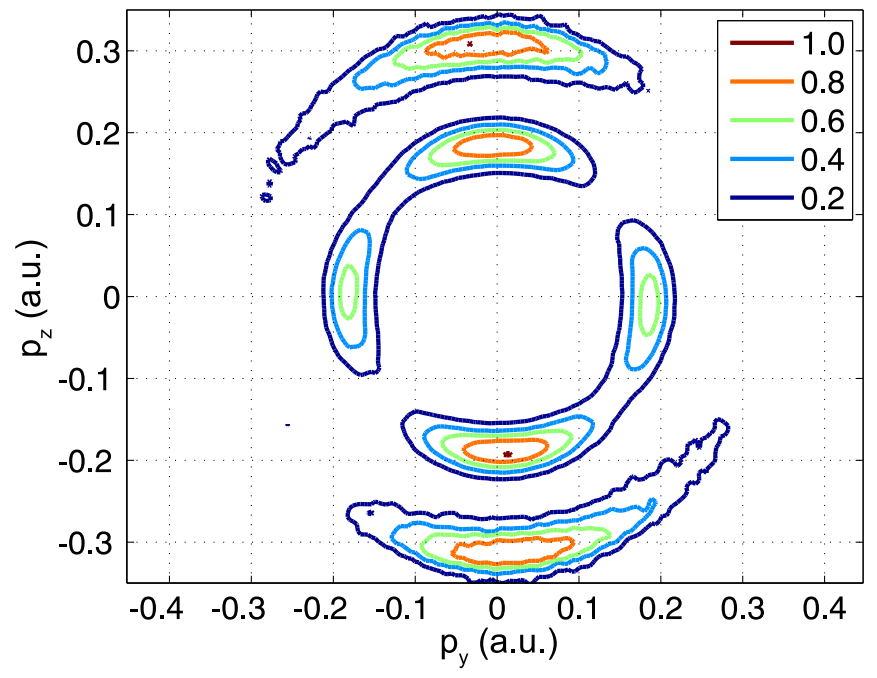

FIG. 10. Contour plot of the relative experimental photoelectron momentum distribution in the central polarization plane obtained for two- and three-photon detachment of $\mathrm{Ag}^{-}$at a wavelength of $1310 \mathrm{~nm}$. The two- and three-photon processes have been individually normalized.

Fig. 11 shows the ellipse representing the rotational direction and magnitude of the laser field for $\varepsilon=0.21$.

\section{DISCUSSION}

One advantage of using the IRT instead of the IAT to recreate the electron distribution, even in the case of an experiment using linear laser polarization, is that the latter introduces distortions along the polarization axis. The IAT is highly sensitive to the definition of the image center. For a

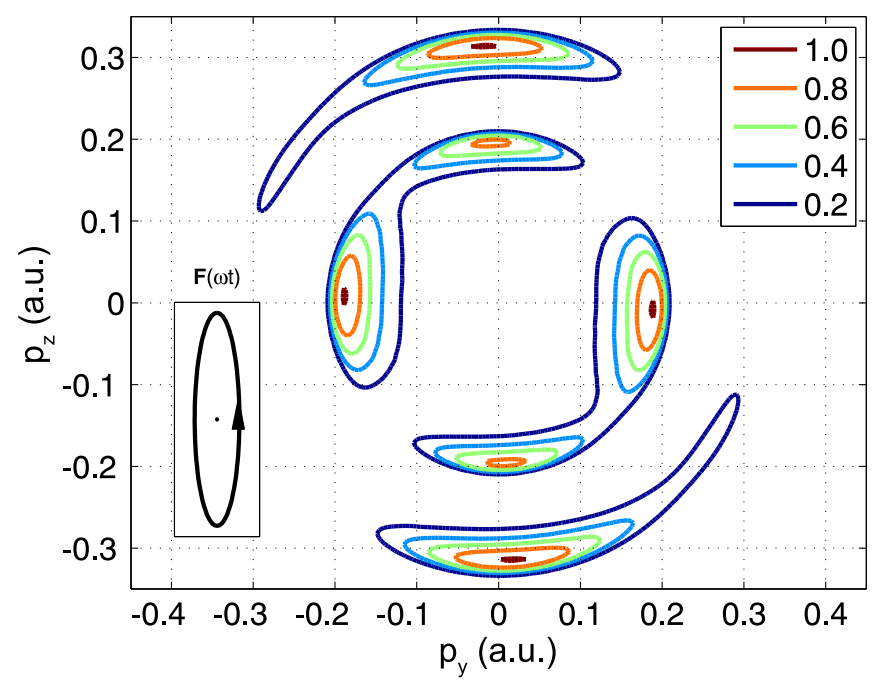

FIG. 11. Contour plot of the relative simulated photoelectron momentum distribution in the central polarization plane obtained through QQES simulation of two- and three-photon detachment of $\mathrm{Ag}^{-}$at a wavelength of $1310 \mathrm{~nm}$. The two- and three-photon processes have been individually normalized. The inset ellipse represents the rotational direction and magnitude of an elliptically polarized laser field with ellipticity $\varepsilon=0.21$. strong-field experiment, it is in general the case that the area of interest lies along the polarization axis. In Refs. [19,20], for instance, we refrained from performing the Abel inversion because of the distortions it might introduce. The IRT, on the other hand, is completely insensitive to the centering of the image in the $x$ direction (parallel to the laser propagation axis). It is instead sensitive to the centering of $w$ (perpendicular to the laser propagation axis). In the case of a strong signal along the linear polarization axis ( $z$ axis), however, the centering of $w$ can be done with higher precision than centering $x$. (For a description of the mentioned coordinate axes, see Fig. 1.)

Another advantage of the tomographic method is the insensitivity of the polarization axis being parallel to the detector plane. When IAT is to be performed, one needs to make sure that the polarization and the detector are perfectly parallel to each other. If not, information about the electron distribution will be inaccurate. In the case of IRT, however, having the polarization axis misaligned merely introduces an offset in the angle $\vartheta$ which can be easily accounted for in postprocessing.

By spreading the information of the electron swarm over a larger area on the detector, the tomographic method is also less sensitive to inhomogeneities in the gain of the detector. Consider a portion of the electron swarm occupying a sphere with volume $V=4 \pi(\Delta p)^{3} / 3$ in momentum space. If the time of flight for the electrons in the EIS is assumed to be $\Delta t$, then, in the case that the polarization orientation is stationary (as in IAT), the electrons will be projected onto an area $\pi(\Delta t \Delta p)^{2}$ on the detector. Let $p_{r}$ be the distance from the axis of rotation to the center of the volume $V$. Now if we instead rotate the polarization axis in small steps, the volume $V$ will sweep out a torus with major radius $p_{r}$. This torus projects onto an area on the detector surface that is $\pi(\Delta t \Delta p)^{2}+2 p_{r} \Delta t \Delta p$. That is to say, we form a weighted average over different positions on the detector to reconstruct the electron distribution, with the effect being most pronounced for large values of $p_{r}$.

\section{CONCLUSION}

We have implemented a tomographic imaging method to recreate the full three-dimensional distribution of photoelectrons produced in a strong-field photodetachment process. The method is general and can be applied to any type of polarization. The tomographic methods stands in contrast to traditional imaging inversion methods, such as Abel inversion, which require prior knowledge of the symmetries of the electron distribution. This limits experiments to linear or circular polarizations. The method developed is also useful in a situation where linear polarization is used, since it allows us to automatically compensate for inhomogeneities in the detector by spreading the information on a larger detector surface. In addition to this, it facilitates a method for detecting polarization defects.

Measurements were made for photodetachment of $\mathrm{Ag}^{-}$ at laser wavelengths of 1310 and $2055 \mathrm{~nm}$. Studying photoelectron emission using negative ions has the advantage that many negative ions, such as $\mathrm{Ag}^{-}$, have a singly bound state [37], making the initial state in the process unambiguously determined. Further, electron affinities are typically much smaller as compared with ionization potentials, making multiphoton detachment accessible with very few photons using 
powerful lasers available in the near-infrared wavelength region. The experimental results were found to agree well with simulations of theoretical models. The results obtained at the wavelength of $1310 \mathrm{~nm}$ revealed an unexpected asymmetry in the plane in which the laser polarization axis is rotated. A range of possible causes for this observation were examined in detail but could be ruled out. Using a QQES model, a residual elliptical polarization with $\varepsilon=0.21 \pm 0.01$ can, however, consistently explain the asymmetry. The present paper is a proof-of-concept study to check the feasibility and usefulness of using tomographic reconstruction in a fast beam with the relatively low count rate of a photodetachment experiment. Our results show that tomography is indeed a powerful method that has the potential of showing details that would otherwise not be visible.

\section{ACKNOWLEDGMENTS}

This work was funded by the the Swedish Research Council Grant No. 2016-0365, the EU-ITN Network ICONIC 238671 and Deutsche Forschungsgemeinschaft (DFG), Grant No. KI 865/3-1. The Authors of Ref. [33] are acknowledged for providing us with the code for calculating the quasienergy and angular distribution using the QQES.
[1] H. Helm, N. Bjerre, M. J. Dyer, D. L. Huestis, and M. Saeed, Images of Photoelectrons Formed in Intense Laser Fields, Phys. Rev. Lett. 70, 3221 (1993).

[2] J. Ullrich, R. Moshammer, A. N. Perumal, and R. Moshammer, Reports on progress in physics related content recoil-ion and electron momentum spectroscopy: Recoil-ion and electron momentum spectroscopy, Rep. Prog. Phys. 66, 1463 (2003).

[3] A. T. J. B. Eppink and D. H. Parker, Velocity map imaging of ions and electrons using electrostatic lenses: Application in photoelectron and photofragment ion imaging of molecular oxygen, Rev. Sci. Instrum. 68, 3477 (1997).

[4] C. Bordas, F. Paulig, H. Helm, and D. L. Huestis, Photoelectron imaging spectrometry: Principle and inversion method, Rev. Sci. Instrum. 67, 2257 (1996).

[5] K. L. Reid, Photoelectron angular distributions, Annu. Rev. Phys. Chem. 54, 397 (2003).

[6] M. Saeed, M. J. Dyer, and H. Helm, Spatial anisotropy of the velocity of electrons emitted from a short-pulse laser focus, Phys. Rev. A 49, 1491 (1994).

[7] T. Wilbois, Single ionization of molecular hydrogen in strong laser fields: Experimental imaging and analysis, Ph.D. dissertation, University of Freiburg, Fakultät für Mathematik und Physik, 2011.

[8] B. J. Whitaker, Imaging in Molecular Dynamics: Technology and Applications, 1st ed. (Cambridge University Press, Cambridge, UK, 2003).

[9] V. Dribinski, A. Ossadtchi, V. A. Mandelshtam, and H. Reisler, Reconstruction of Abel-transformable images: The Gaussian basis-set expansion Abel transform method, Rev. Sci. Instrum. 73, 2634 (2002).

[10] G. A. Garcia, L. Nahon, and I. Powis, Two-dimensional charged particle image inversion using a polar basis function expansion, Rev. Sci. Instrum. 75, 4989 (2004).

[11] S. Kim and A. K. Khambampati, Mathematical concepts for image reconstruction in tomography, in Industrial Tomography, edited by M. Wang, Woodhead Publishing Series in Electronic and Optical Materials (Woodhead Publishing, Sawston, UK, 2015), pp. 305-346.

[12] C. Haisch, Optical tomography, Annu. Rev. Anal. Chem. 5, 57 (2012).

[13] J. Itatani, J. Levesque, D. Zeidler, H. Niikura, H. Pépin, J. C. Kieffer, P. B. Corkum, and D. M. Villeneuve, Tomographic imaging of molecular orbitals, Nature 5, 867 (2004).

[14] M. Wollenhaupt, M. Krug, J. Köhler, T. Bayer, C. SarpeTudoran, and T. Baumert, Three-dimensional tomographic reconstruction of ultrashort free electron wave packets, Appl. Phys. B 95, 647 (2009).

[15] C. Smeenk, L. Arissian, A. Staudte, D. M. Villeneuve, and P. B. Corkum, Momentum space tomographic imaging of photoelectrons, J. Phys. B: At. Mol. Opt. Phys. 42, 185402 (2009).

[16] M. Wollenhaupt, C. Lux, M. Krug, and T. Baumert, Tomographic reconstruction of designer free-electron wave packets, ChemPhysChem 14, 1341 (2013).

[17] P. Hockett, M. Staniforth, and K. L. Reid, Photoelectron angular distributions from rotationally state-selected $\mathrm{NH}_{3}\left(\mathrm{~B}^{1} E^{\prime \prime}\right)$ : Dependence on ion rotational state and polarization geometry, Mol. Phys. 108, 1045 (2010).

[18] P. Hockett, C. Lux, M. Wollenhaupt, and T. Baumert, Maximum-information photoelectron metrology, Phys. Rev. A 92, 013412 (2015).

[19] H. Hultgren, M. Eklund, D. Hanstorp, and I. Yu. Kiyan, Electron dynamics in the ground state of a laser-generated carbon atom, Phys. Rev. A 87, 031404(R) (2013).

[20] M. Eklund, H. Hultgren, D. Hanstorp, and I. Yu. Kiyan, Orbital alignment in atoms generated by photodetachment in a strong laser field, Phys. Rev. A 88, 023423 (2013).

[21] R. Reichle, H. Helm, and I. Yu. Kiyan, Detailed comparison of theory and experiment of strong-field photodetachment of the negative hydrogen ion, Phys. Rev. A 68, 063404 (2003).

[22] Y. Nievergelt, Elementary inversion of Radon's transform, SIAM Rev. 28, 79 (1986).

[23] R. N. Bracewell, Strip integration in radio astronomy, Aust. J. Phys. 9, 198 (1956).

[24] R. Bracewell, Numerical transforms, Science 248, 697 (1990).

[25] https://www.mathworks.com/help/images/ref/iradon.html (2020).

[26] R. C. Bilodeau, M. Scheer, and H. K. Haugen, Infrared laser photodetachment of transition metal negative ions: Studies on $\mathrm{Cr}^{-}, \mathrm{Mo}^{-}, \mathrm{Cu}^{-}$and $\mathrm{Ag}^{-}$, J. Phys. B: At. Mol. Opt. Phys. 31, 3885 (1998).

[27] See Supplemental Material at http://link.aps.org/supplemental/ 10.1103/PhysRevA.102.023114 for Movie 1 shows a series of projections for a full rotation at steps of $3^{\circ}$ for photodetachment at $1310 \mathrm{~nm}$. Four sample projections from the video at angles of $\vartheta=0^{\circ}, 30^{\circ}, 60^{\circ}$, and $90^{\circ}$ are shown in Fig. 2.

[28] See Supplemental Material at http://link.aps.org/supplemental/ 10.1103/PhysRevA.102.023114 for Movie 2 shows a straight projection of the full reconstructed three-dimensional photoelectron momentum distribution for detachment of $\mathrm{Ag}^{-}$at $1310 \mathrm{~nm}$. Projections along the $x$ and $y$ axes from the movie 
are shown in Fig. 3. The movie shows three isosurfaces at values 0.4 (brown), 0.2 (green), and 0.1 (blue) of the maximum photoemission distribution value. The laser is propagating in the $x$ direction and the laser polarization is along the $z$ axis.

[29] See Supplemental Material at http://link.aps.org/supplemental/ 10.1103/PhysRevA.102.023114 for Movie 3 shows a slanted projection of the full reconstructed three-dimensional photoelectron momentum distribution for detachment of $\mathrm{Ag}^{-}$at $1310 \mathrm{~nm}$. Projections along the $x$ and $y$ axes from the movie are shown in Fig. 3. The movie shows three isosurfaces at values 0.4 (brown), 0.2 (green), and 0.1 (blue) of the maximum photoemssion distribution value. The laser is propagating in the $x$ direction and the laser polarization is along the $z$ axis.

[30] See Supplemental Material at http://link.aps.org/supplemental/ 10.1103/PhysRevA.102.023114 for Movie 4 shows a straight projection of the full reconstructed three-dimensional photoelectron momentum distribution for detachment of $\mathrm{Ag}^{-}$at $2055 \mathrm{~nm}$. Projections along the $x$ and $y$ axes from the movie are shown in Fig. 4. The movie shows three isosurfaces at values 0.4 (brown), 0.2 (green), and 0.1 (blue) of the maximum photoemssion distribution value. The laser is propagating in the $x$ direction and the laser polarization is along the $z$ axis.

[31] See Supplemental Material at http://link.aps.org/supplemental/ 10.1103/PhysRevA.102.023114 for Movie 5 shows a slanted projection of the full reconstructed three-dimensional photo- electron momentum distribution for detachment of $\mathrm{Ag}^{-}$at $2055 \mathrm{~nm}$. Projections along the $x$ and $y$ axes from the movie are shown in Fig. 4. The movie shows three isosurfaces at values 0.4 (brown), 0.2 (green), and 0.1 (blue) of the maximum photoemssion distribution value. The laser is propagating in the $x$ direction and the laser polarization is along the $z$ axis.

[32] G. F. Gribakin and M. Yu. Kuchiev, Multiphoton detachment of electrons from negative ions, Phys. Rev. A 55, 3760 (1997).

[33] N. L. Manakov, M. V. Frolov, B. Borca, and A. F. Starace, Multiphoton detachment of a negative ion by an elliptically polarized, monochromatic laser field, J. Phys. B: At. Mol. Opt. Phys. 36, R49 (2003).

[34] B. Bergues, Z. Ansari, D. Hanstorp, and I. Yu. Kiyan, Photodetachment in a strong laser field: An experimental test of keldysh-like theories, Phys. Rev. A 75, 063415 (2007).

[35] H. R. Reiss, Comment on "photodetachment in a strong laser field: An experimental test of keldysh-like theories," Phys. Rev. A 77, 067401 (2008).

[36] B. Bergues, Z. Ansari, D. Hanstorp, and I. Yu. Kiyan, Reply to "Comment on 'Photodetachment in a strong laser field: An experimental test of Keldysh-like theories"', Phys. Rev. A 77, 067402 (2008).

[37] T. Andersen, H. K. Haugen, and H. Hotop, Binding energies in atomic negative ions: III, J. Phys. Chem. Ref. Data 28, 1511 (1999). 